\title{
Gas Discrimination by Simultaneous Sound Velocity and Attenuation Measurements using Uncoated Capacitive Micromachined Ultrasonic Transducers
}

Luis Iglesias Hernandez

University of Bordeaux

Priyadarshini Shanmugam

University of Tours

Jean-Francois Michaud

University of Tours

Daniel Alquier

University of Tours

Dominique Certon

University of Tours

Isabelle Dufour ( $\boldsymbol{Q}$ isabelle.dufour@ims-bordeaux.fr)

University of Bordeaux

\section{Research Article}

Keywords: sensors, poor, applications, uncoated, selectivity

Posted Date: September 8th, 2021

DOI: https://doi.org/10.21203/rs.3.rs-863992/v1

License: (9) (i) This work is licensed under a Creative Commons Attribution 4.0 International License.

Read Full License 


\title{
Gas Discrimination by Simultaneous Sound Velocity and Attenuation Measurements using Uncoated Capacitive Micromachined Ultrasonic Transducers
}

\author{
Luis Iglesias Hernandez ${ }^{1}$, Priyadarshini Shanmugam² ${ }^{2}$, Jean-François Michaud ${ }^{2}$, Daniel \\ Alquier $^{2}$, Dominique Certon ${ }^{2}$, and Isabelle Dufour ${ }^{*}$ \\ ${ }^{1}$ University of Bordeaux, Laboratoire IMS UMR-CNRS 5218, 351 cours de la libération, 33405 TALENCE Cedex, \\ France \\ ${ }^{2}$ University of Tours, GREMAN, UMR-CNRS 7347, 16 rue Pierre et Marie Curie, BP 7155, 37071 TOURS Cedex 2, \\ France \\ *isabelle.dufour@ims-bordeaux.fr
}

\begin{abstract}
Chemically functionalized or coated sensors are by far the most employed solution in gas sensing. However, their poor long term stability represents a concern in applications dealing with hazardous gases. Uncoated sensors are durable but their selectivity is poor or non-existent. In this study, multi-parametric discrimination is used as an alternative to selectivity for uncoated capacitive micromachined ultrasonic transducers (CMUTs). This paper shows how measuring simultaneously the attenuation coefficient and the time of flight under different nitrogen mixtures allows to identify hydrogen, carbon dioxide and methane from each other and determine their concentration along with identification of temperature drift. Theoretical comparison and specific signal processing to deal with the issue of multiple reflections are also presented. Some potential applications are monitoring of refueling stations, vehicles and nuclear waste storage facilities.
\end{abstract}

\section{Introduction}

Nowadays, most gas sensors present a chemical component such as a functionalized coating specifically engineered to target a particular analyte ${ }^{1,2}$. However, it is well known that such chemical coating presents long term stability issues ${ }^{3}$. This results in the need for recalibrating the sensor, typically every couple of months. For applications dealing with hazardous gases such as hydrogen, $\mathrm{H}_{2}$, or methane, $\mathrm{CH}_{4}$, this becomes dangerous for the operators. Alternatively, it is possible to compensate this drift digitally, for instance, with artificial intelligence ${ }^{4}$ or sophisticated models ${ }^{5}$. Nevertheless, this naturally increases both the manufacturing and the development cost. For this reason and despite their typically lower selectivity and worse limits of detection $(\mathrm{LOD})^{2}$, the development of uncoated gas sensors has increased in popularity over the past decade. Indeed, some parameters such as the resonant frequency of a cantilever ${ }^{6}$, the gas sound velocity ${ }^{7}$ and the acoustic attenuation coefficient ${ }^{8,9}$ depend on the gas physical properties. This allowed the development of gas density sensors ${ }^{10}$ and in some cases these principles can be exploited to measure a binary gas mixture concentration ${ }^{11,12}$ including some consisting of capacitive micromachined ultrasonic transducers (CMUTs) ${ }^{13}$. A typical LOD associated to uncoated sensors is about few percents down to 100ppm and they are usually considered as non selective. However, in some specific cases, uncoated sensors can show performances close to coated sensors such as a LOD of a few $\mathrm{ppm}^{14}$ and even selectivities higher than $10^{15}$. In order to overcome the problem of selectivity, an alternative consists in discrimination by measuring multiple physical properties of a gas mixture such as mass density and viscosity ${ }^{16}$. In this paper, we propose to measure simultaneously two other gas properties; the acoustic attenuation coefficient, $\alpha$, for frequencies ranging from $1 \mathrm{MHz}$ to $4.5 \mathrm{MHz}$, and the time of flight, $\tau$, which is the time taken by an acoustic ultrasonic wave to travel from an emitter to a receiver passing through the gas to be characterized. In such a setup, $\alpha$ can be defined as:

$$
\alpha=\frac{1}{d} \ln \frac{P_{e}}{P_{r}}
$$

where $d$ is the distance between the emitter and the receiver and $P_{e}$ and $P_{r}$ are the amplitudes of the acoustic wave at the emission and the reception ends, respectively. This work is the continuation of previous work showing the possibility to use CMUTs to determine the concentration of a binary gas mixture by measuring either $\tau^{17,18}$ or $\alpha^{15}$ separately and in which the best selectivity obtained was 12 . Although this value is high enough for some applications, it can be considered very low for 
others. For this reason, a setup capable of measuring both measurements is presented in this study. The choice of using CMUTs comes from several advantages over other ultrasonic transducers such as piezoelectric based ultrasonic transducers. These are easier to integrate and present a wider bandwidth, which allows to perform gas measurements over a larger part of the attenuation spectrum ${ }^{19}$. Sec. 2 shows characterization of the sensor in terms of temperature influence and sensitivity for three mixtures of nitrogen, $\mathrm{N}_{2}$, with either hydrogen, $\mathrm{H}_{2}$, carbon dioxide, $\mathrm{CO}_{2}$, or methane, $\mathrm{CH}_{4}$. The choice of $\mathrm{N}_{2}$ as the main gas is done in order to simplify the modeling and due to its similarity to air in terms of acoustical properties. In Sec. 3, the advantages of measuring simultaneously $\tau$ and $\alpha$, based on the results, are discussed. Finally, in Sec. 4, details are given about the setup such as the microfabrication of the CMUTs and the signal processing employed for a robust measurement, even in case of spurious reflections and electrical coupling between the emitter and the receiver.

\section{Results}

Details on how both the time of flight, $\tau$, and the shift in attenuation with respect to pure nitrogen at $20^{\circ} \mathrm{C}, \Delta \alpha$, are measured can be found in Sec. 4 along with detailed information about the setup and the CMUT arrays.

\subsection{Temperature Characterization}

This section seeks to estimate the sensor cross sensitivity to the temperature, $T$, of both the measurement of $\tau, S_{\tau}^{T}$, and of $\Delta \alpha$, $S_{\alpha}^{T}$ between $20^{\circ} \mathrm{C}$ and $50^{\circ} \mathrm{C}$. In order to achieve this, both $\tau$ and $\Delta \alpha$ where measured at different temperatures under pure $\mathrm{N}_{2}$ in that temperature range. The resulting characteristics are shown in Fig. 1. In the case of the measurement of $\tau$, the theoretical
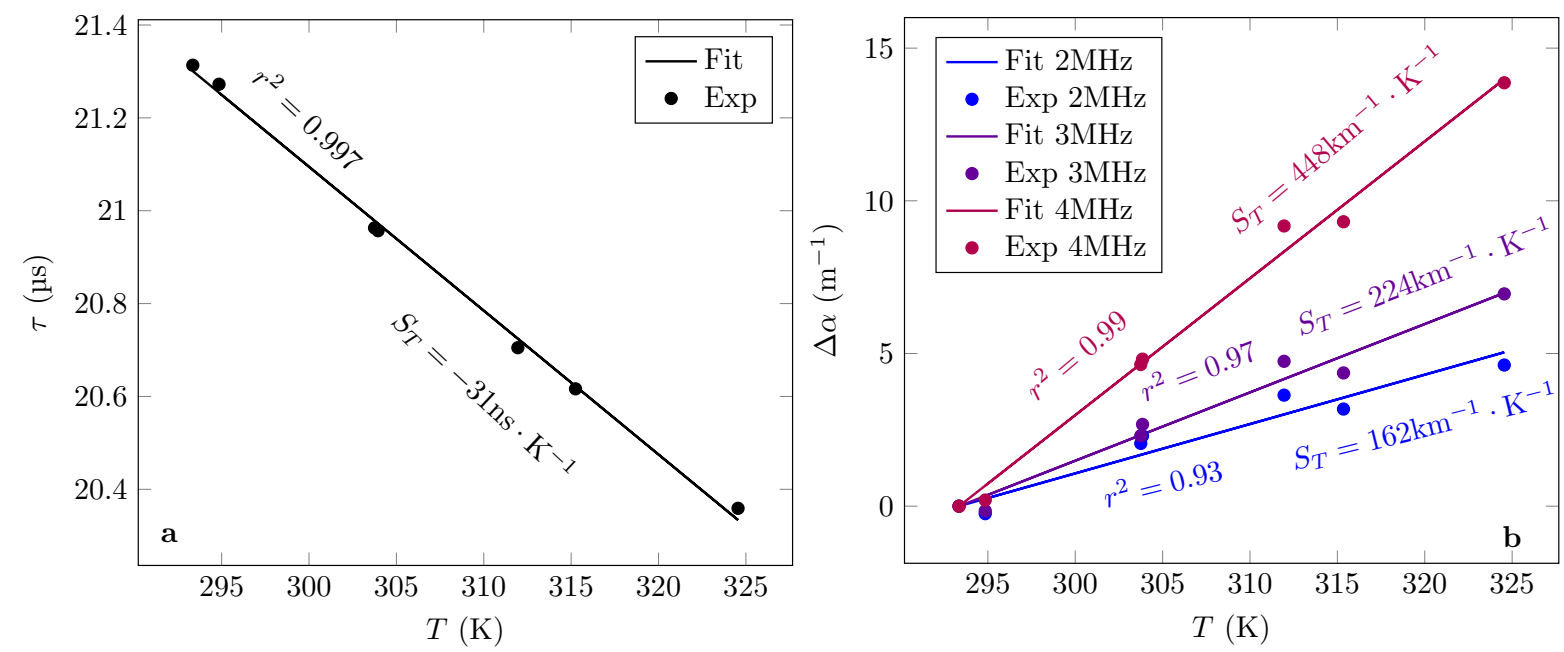

Figure 1. Sensor response to temperature under pure $\mathrm{N}_{2}$. (a) Time of flight. (b) Attenuation shift.

value can be estimated for an ideal gas by exploiting Newton-Laplace formula for the sound velocity, $v$, of an ideal gas ${ }^{7}$ :

$$
v=\sqrt{\frac{\gamma R T}{M}},
$$

where $\gamma$ is the heat capacities ratio (considered constant in this range of temperature) and $M$ the molar mass of the gas. Differentiating Eq. 2 results in:

$$
\frac{\Delta v}{v}=\frac{1}{2} \frac{\Delta T}{T},
$$

where $\Delta v$ and $\Delta T$ are small variations of $v$ and $T$, respectively. Since $v=d / \tau$,

$$
\frac{\Delta v}{v}=-\frac{\Delta \tau}{\tau},
$$

where $\Delta \tau$ represents small variations of $\tau$. Finally, $S_{\tau}^{T}$ is given by:

$$
S_{\tau}^{T}=\frac{\Delta \tau}{\Delta T}=-\frac{1}{2} \frac{\tau}{T}
$$


For pure nitrogen at $20^{\circ} \mathrm{C}, \tau=21.35 \mu$ s (distance $d \approx 7.5 \mathrm{~mm}$ ), the theoretical value is $S_{\tau}^{T}=-36 \mathrm{~ns} / \mathrm{K}$ against the experimental value of $-31 \mathrm{~ns} / \mathrm{K}$. The difference might come from the relatively large temperature variation range or the fact that the sensor and the thermocouple used to measure the temperature are a few centimeters apart inside the oven. However, as it will be shown later, this difference is sufficiently small for the purpose of this article. In the case of $\Delta \alpha$, due to the complexity of its analytical expression and its dependence on an important number of the gas properties, the temperature influence on the attenuation in this study is purely experimental. From Fig. 1, it can be concluded that, in the range between $2 \mathrm{MHz}$ and $4 \mathrm{MHz}$, the sensor's temperature cross-sensitivity increases with the frequency and its value will be exploited in the following section.

\subsection{Gas Characterization}

The temperature influence on the sensor being known, this section aims to characterize the sensor response to different gas mixtures. For that, the sensor was exposed to sequences where pure $\mathrm{N}_{2}$ is alternated with binary mixtures of $\mathrm{N}_{2}$ with either $\mathrm{H}_{2}$, $\mathrm{CO}_{2}$ or $\mathrm{CH}_{4}$ at different molar concentrations, $x$ of the targeted gas. An example of the measurements at $4 \mathrm{MHz}$ is shown in Fig. 2 for both,(a) the shift in time of flight with respect to $\mathrm{N}_{2}, \Delta \tau$, and (b) $\Delta \alpha$. From these measurements one can extract the sensitivities through calibration curves, both of $\Delta \tau$ (c) and $\Delta \alpha$ (d) to the targeted gas $\left(\mathrm{H}_{2}, \mathrm{CO}_{2}\right.$ or $\left.\mathrm{CH}_{4}\right), S_{\tau}$ and $S_{\alpha}$, respectively.

Since $S_{\alpha}$ depends on the excitation frequency, it was measured for the whole frequency range of $1 \mathrm{MHz}$ up to $4.5 \mathrm{MHz}$ (e) which is limited by the charge amplifier at the lower end and by the strong attenuation above $4.5 \mathrm{MHz}$. Similar to the derivation of the expression of the temperature cross-sensitivity, the expression of $S_{\tau}$ can be obtained ${ }^{17}$ :

$$
S_{\tau}=\frac{\Delta \tau}{x}=\frac{\tau}{2}\left[\frac{\rho^{G}}{\rho^{N 2}}+\frac{c_{v}^{G}}{c_{v}^{N 2}}-\frac{c_{p}^{G}}{c_{p}^{N 2}}-1\right],
$$

where $c_{p}^{G}$ and $c_{v}^{G}$ are the isobaric and isochoric heat capacities per unit mass of the targeted pure gas, respectively, and $c_{p}^{N 2}$ and $c_{v}^{N 2}$ the ones of pure $\mathrm{N}_{2}$. To derive an exact expression of $S_{\alpha}$ would be unnecessarily complex. Therefore, in this study, a simple model is used from the expression of the attenuation of a pure gas $\alpha_{p}{ }^{20}$ :

$$
\alpha_{p}=\frac{2 \pi^{2} f^{2}}{\rho v^{3}}\left(\frac{4}{3} \eta+\eta_{v}+\frac{\gamma-1}{c_{p}} K\right)+\alpha_{v r}
$$

where $\eta$ is the viscosity of the gas, $\eta_{v}$ its volume viscosity, $K$ its thermal conductivity, $c_{p}$ its isobaric heat capacity per unit mass and $\alpha_{v r}$ the vibrational relaxation attenuation contribution modeled $\mathrm{as}^{21}$ :

$$
\alpha_{v r}=\frac{A f}{v} \frac{f / f_{v r}}{1+f^{2} / f_{v r}^{2}}
$$

where $A$ is a constant depending on the strength of the relaxation process and $f_{v r}$ the frequency corresponding to the maximum of the normalized attenuation coefficient $\alpha v / f$. When dealing with a binary gas mixture, an additional contribution of the diffusion process $\alpha_{\delta}$ is needed, especially for molecules with very different molar masses $M$ such as $\mathrm{H}_{2}$ and $\mathrm{N}_{2}{ }^{22}$

$$
\alpha_{\delta}=\frac{2 \pi^{2} f^{2}}{\rho v^{3}} x(1-x) \gamma \delta\left(\frac{\Delta M}{M}+\frac{\gamma-1}{\gamma} a\right)^{2},
$$

where $a$ is the thermal diffusion factor of the mixture, $\delta$ its diffusion coefficient and $\Delta M$ is the difference in molar masses between both constituents of the mixture. The shift in attenuation with respect to $\mathrm{N}_{2}$ is then approximated to:

$$
\Delta \alpha=\left[x \alpha_{G}+(1-x) \alpha_{N 2}+\alpha_{\delta}\right]-\alpha_{N 2},
$$

where $\alpha_{N 2}$ and $\alpha_{G}$ are the attenuation coefficients in the pure state of $\mathrm{N}_{2}$ and the targeted gas respectively, as given by Eq. 7. Finally, $S_{\alpha}$ is simply $\Delta \alpha(x=1 \%)$. All the necessary constants to compute both $S_{\tau}$ and $S_{\alpha}$ for the three mixtures studied in this article are given in Table 1. From them the theoretical values of $S_{\tau}$ for the mixtures $\mathrm{N}_{2}-\mathrm{H}_{2} \mathrm{~N}_{2}-\mathrm{CO}_{2}$ and $\mathrm{N}_{2}-\mathrm{CH}_{4}$ ) are $-100 \mathrm{~ns} / \%$, $72 \mathrm{~ns} / \%$ and $-35 \mathrm{~ns} / \%$, respectively. The measurements (Fig. 2.c) are consistent with the theory. Additionally, in Fig. 2.e, the theoretical values of $S_{\alpha}$ are displayed in dashed lines along with the measured results. Once again the results are consistent with the theory in terms of sign and the overall form of the variation. In the case of $\mathrm{H}_{2}$, there seems to be some additional losses than the ones predicted by the linear model from Eq. 10. This is due to the beginning of the relaxation attenuation peak of $\mathrm{H}_{2}$, which is already an important and non-linear component of the attenuation ${ }^{8}$. Nevertheless, in a practical application, this can be solved with a calibration mapping $\Delta \alpha$ to the molar fraction of $\mathrm{H}_{2}$. 

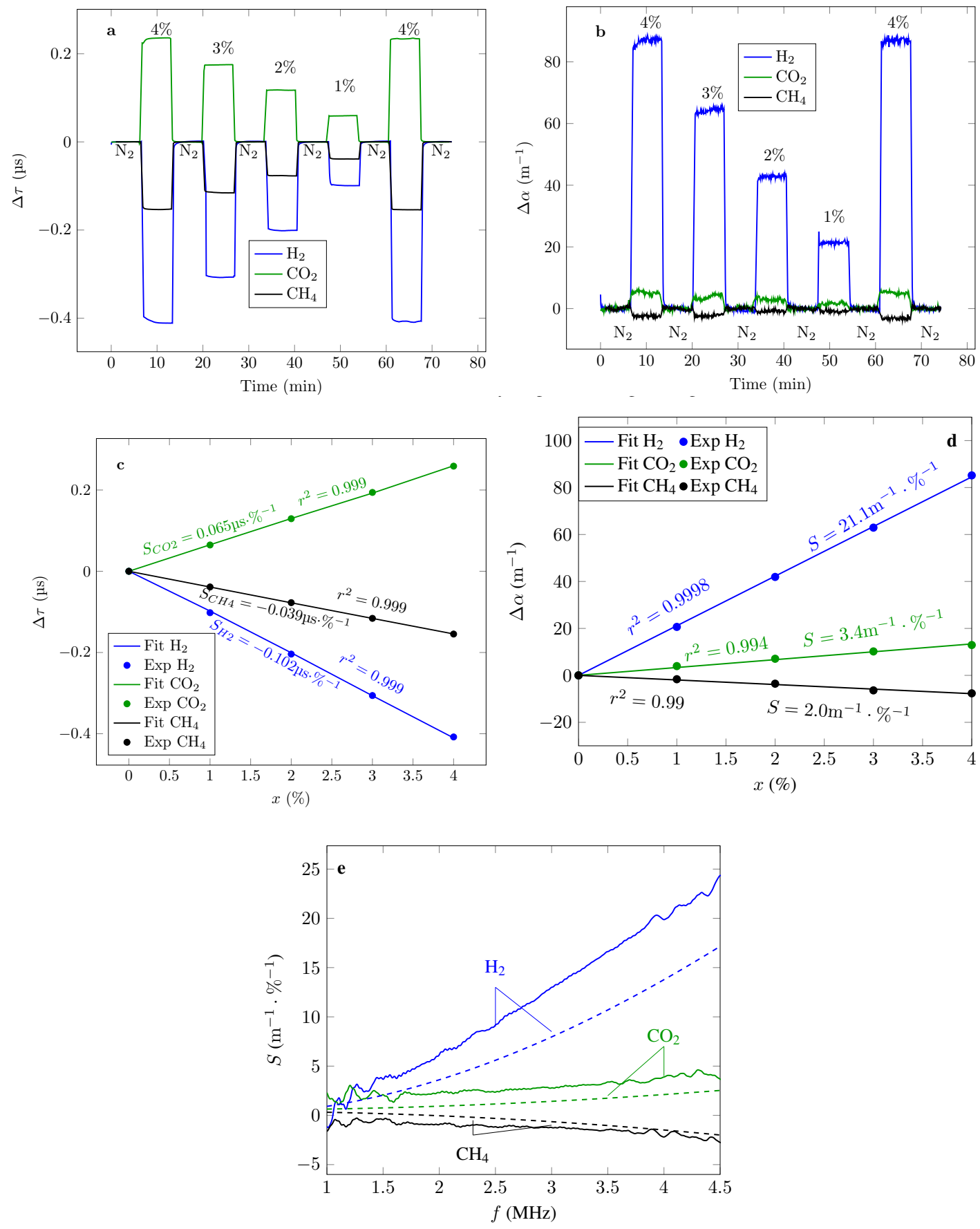

Figure 2. Sensor response to binary mixtures of $\mathrm{N}_{2}$ with either $\mathrm{H}_{2}, \mathrm{CO}_{2}$ or $\mathrm{CH}_{4}$. (a) Shift in time of flight when alternating the mixture at different concentrations with pure $\mathrm{N}_{2}$. (b) Attenuation shift at $4 \mathrm{MHz}$ when alternating the mixture at different concentrations with pure $\mathrm{N}_{2}$. (c) Calibration curves from (a). (d) Calibration curves from (b). (e) Experimental (solid lines) and theoretical (dashed lines) values of the sensor sensitivity in attenuation shift as a function of the excitation frequency for the three binary gas mixtures.

\subsection{Discrimination}

So far, the measurements of $\Delta \tau$ and $\Delta \alpha$ have been displayed separately. However they are always measured simultaneously. Therefore, one can exploit this in order to identify the different mixtures. Indeed, as it is shown in Fig. 3.a, in theory (Eqs. 6 and 10 ), when plot $\Delta \alpha$ against $\Delta \tau$, the three different types of mixtures are placed in different parts of the plane. Additionally, since 


\begin{tabular}{lcccc}
\hline Property & $\mathrm{N}_{2}$ & $\mathrm{H}_{2}$ & $\mathrm{CO}_{2}$ & $\mathrm{CH}_{4}$ \\
\hline$\rho\left(\mathrm{kg} / \mathrm{m}^{3}\right)$ & $1.16^{24}$ & $0.0827^{24}$ & $1.82^{24}$ & $0.68^{24}$ \\
\hline$\eta(\mu \mathrm{Pa} . \mathrm{s})$ & $17.6^{24}$ & $8.8^{24}$ & $14.7^{24}$ & $11.0^{24}$ \\
\hline$\eta_{v}(\mu \mathrm{Pa} . \mathrm{s})$ & $12.8^{25}$ & $265^{25}$ & $5.4^{26}$ & $14.5^{25}$ \\
\hline$K(\mathrm{~W} / \mathrm{K} / \mathrm{m})$ & $0.024^{24}$ & $0.168^{24}$ & $0.015^{24}$ & $0.030^{24}$ \\
\hline$c_{p}(\mathrm{~kJ} / \mathrm{K} / \mathrm{kg})$ & $1.04^{24}$ & $14.3^{24}$ & $0.844^{24}$ & $2.22^{24}$ \\
\hline$c_{v}(\mathrm{~kJ} / \mathrm{K} / \mathrm{kg})$ & $0.743^{24}$ & $10.2^{24}$ & $0.655^{24}$ & $1.70^{24}$ \\
\hline$\gamma(\mathrm{x} 1)$ & $1.4^{24}$ & $1.4^{24}$ & $1.3^{24}$ & $1.3^{24}$ \\
\hline$v(\mathrm{~m} / \mathrm{s})$ & $349^{24}$ & $1306^{24}$ & $267^{24}$ & $445^{24}$ \\
\hline$f_{v r}$ & $0.01 \mathrm{~Hz}^{27}$ & $13 \mathrm{MHz}^{28}$ & $30 \mathrm{kHz}^{8}$ & $150 \mathrm{kHz}^{8}$ \\
\hline$A(\mathrm{x} 1)$ & $6 \mathrm{x} 10^{-427}$ & $0.56^{28}$ & $0.24^{8}$ & $6.6 \times 10^{-28}$ \\
\hline$M(\mathrm{~g} / \mathrm{mol})$ & $28^{24}$ & $2^{24}$ & $44^{24}$ & $16^{24}$ \\
\hline$\delta(\mathrm{cm} / \mathrm{s})$ & $\mathrm{NA}$ & $0.77^{29}$ & $0.15^{29}$ & $0.22^{29}$ \\
\hline$a(\mathrm{x} 1)$ & $\mathrm{NA}$ & $0.26^{30}$ & $0.065^{31}$ & $0.070^{32}$ \\
\hline & & & &
\end{tabular}

Table 1. Physical properties of gases
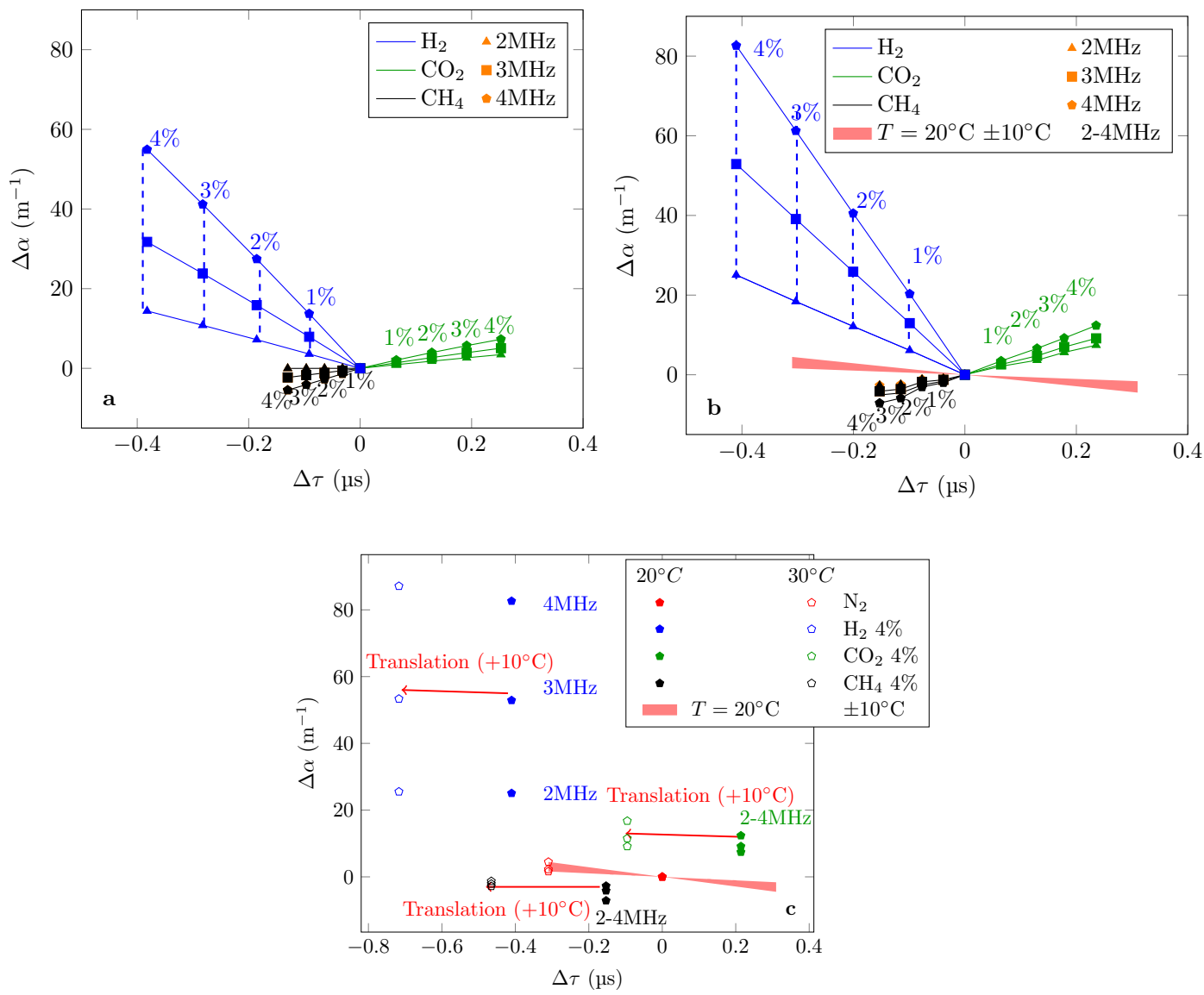

Figure 3. $\Delta \alpha$ as a function of $\Delta \tau$ for different binary gas mixtures. (a) Theoretical values. (b) Measurements. (c) Influence of the temperature on the discrimination resulting in a translation effect.

in absolute value, $S_{\alpha}$ increases with the frequency, it is easier to distinguish between them at higher frequencies (4MHz rather than at $2 \mathrm{MHz}$ ). Fig. 3.b shows the corresponding measurements resulted from the previous characterization as well as the influence of the temperature, taking into account the thermal cross-sensitivities measured in Sec. 2.1. Additional measurements detecting $4 \%$ of each of the three mixtures $\left(\mathrm{N}_{2}\right.$ with either $\mathrm{H}_{2}, \mathrm{CO}_{2}$ or $\left.\mathrm{CH}_{4}\right)$ at $30^{\circ} \mathrm{C}$ and at 1,2 and $3 \mathrm{MHz}$ were performed and 
displayed with the corresponding measurements at $20^{\circ} \mathrm{C}$ in Fig.3.c. From them it can be observed that the main influence of the temperature is an horizontal displacement (shift in $\tau$ ) with a relatively small displacement along the axis of $\Delta \alpha$.

\section{Discussion}

The CMUT ultrasonic gas sensor presented in this paper allows to measure simultaneously both the time of flight and the attenuation coefficient in a binary gas mixture. This allows to discriminate, at a fixed temperature, between the proposed binary mixtures of $\mathrm{N}_{2}$ with either $\mathrm{H}_{2}, \mathrm{CO}_{2}$ or $\mathrm{CH}_{4}$. Remarkably, a change in the sensor output due to the temperature can be distinguished by a small change in the attenuation coefficient and a large shift in the time of flight. This means that, to some extent the sensor presents also temperature sensor capabilities that can help for numerical compensation. This can be exploited even further by the fact that a slight increase in $T$ from $20^{\circ} \mathrm{C}$ to $30^{\circ} \mathrm{C}$ results mainly in a substantial decrease in $\tau$ with a small shift in $\Delta \alpha$. This means that if a slight loss in accuracy (due to the minor distortion in attenuation caused by the temperature) can be afforded, whenever a change in $T$ is detected (delimited by the red zone in Fig. 3.b), the sensor can perform a self recalibration compensating such effect. Additionally, after the mixture is identified, the concentration can be determined with calibration curves. Concretely, the simultaneous measurement of time of flight and attenuation coefficient with CMUTs results in a robust three binary gas mixtures sensor with built in temperature sensor capabilities and long term stability.

\section{Methods}

\subsection{CMUT Details}

An optical microscope picture of the CMUT array used in this study is shown in Fig. 4 along with 3D schematics from different perspectives of single CMUT. The microfabrication protocol used is similar to the one presented in ${ }^{23}$. Their geometrical

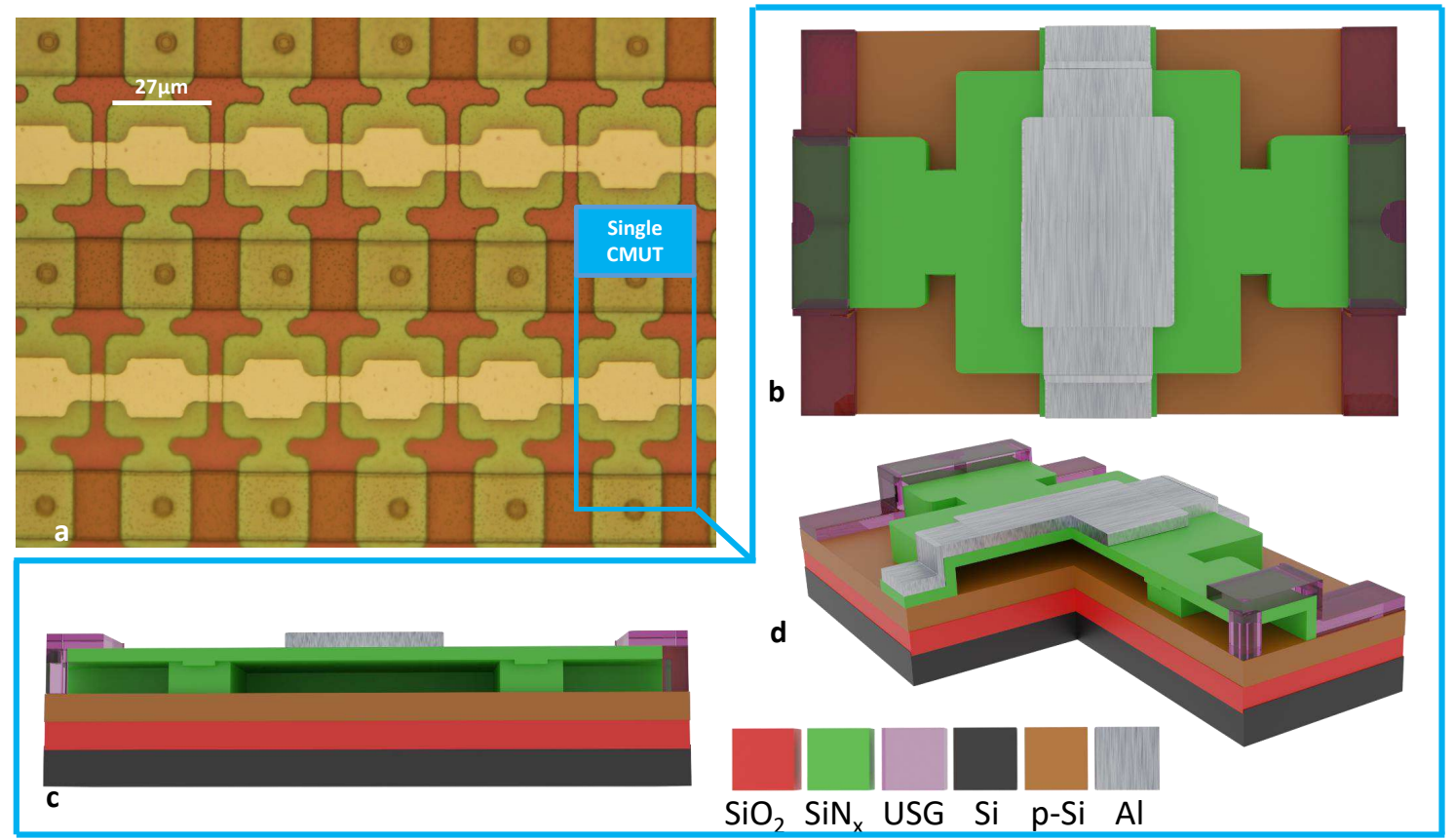

Figure 4. Sensor used in this study. (a) Optical microscope image showing part of the CMUT array. (b) (c) and (d) 3D sketch showing the top view, a transversal cut and a partial cut respectively, where USG stands for Undoped Silicon Glass. Sketch not to scale for visualization purposes.

characteristics along with polarization and excitation details are given in Tab. 2.

\subsection{Setup}

The setup to measure both the shift in attenuation, $\Delta \alpha$, and the sound velocity, $v$, for different gases at different concentrations is shown in Fig. 5. The gases from industrial grade bottles (1) are mixed by a system of flowmeters (2) controlled by a computer 


\begin{tabular}{cc}
\hline Membrane Size $(\mu \mathrm{mx} \mu \mathrm{m})$ & $27 \times 27$ \\
\hline Gap $(\mathrm{nm})$ & 250 \\
\hline Collapse Voltage $(\mathrm{V})$ & 98 \\
\hline Reception DC Voltage $(\mathrm{V})$ & 70 \\
\hline Emission DC Voltage $(\mathrm{V})$ & 50 \\
\hline Emission AC Voltage Peak to Peak $(\mathrm{V})$ & 40 \\
\hline Resonant Frequency $(\mathrm{MHz})$ & 9.6 \\
\hline Quality Factor $(\mathrm{x} 1)$ & 30 \\
\hline
\end{tabular}

Table 2. CMUTs characteristics

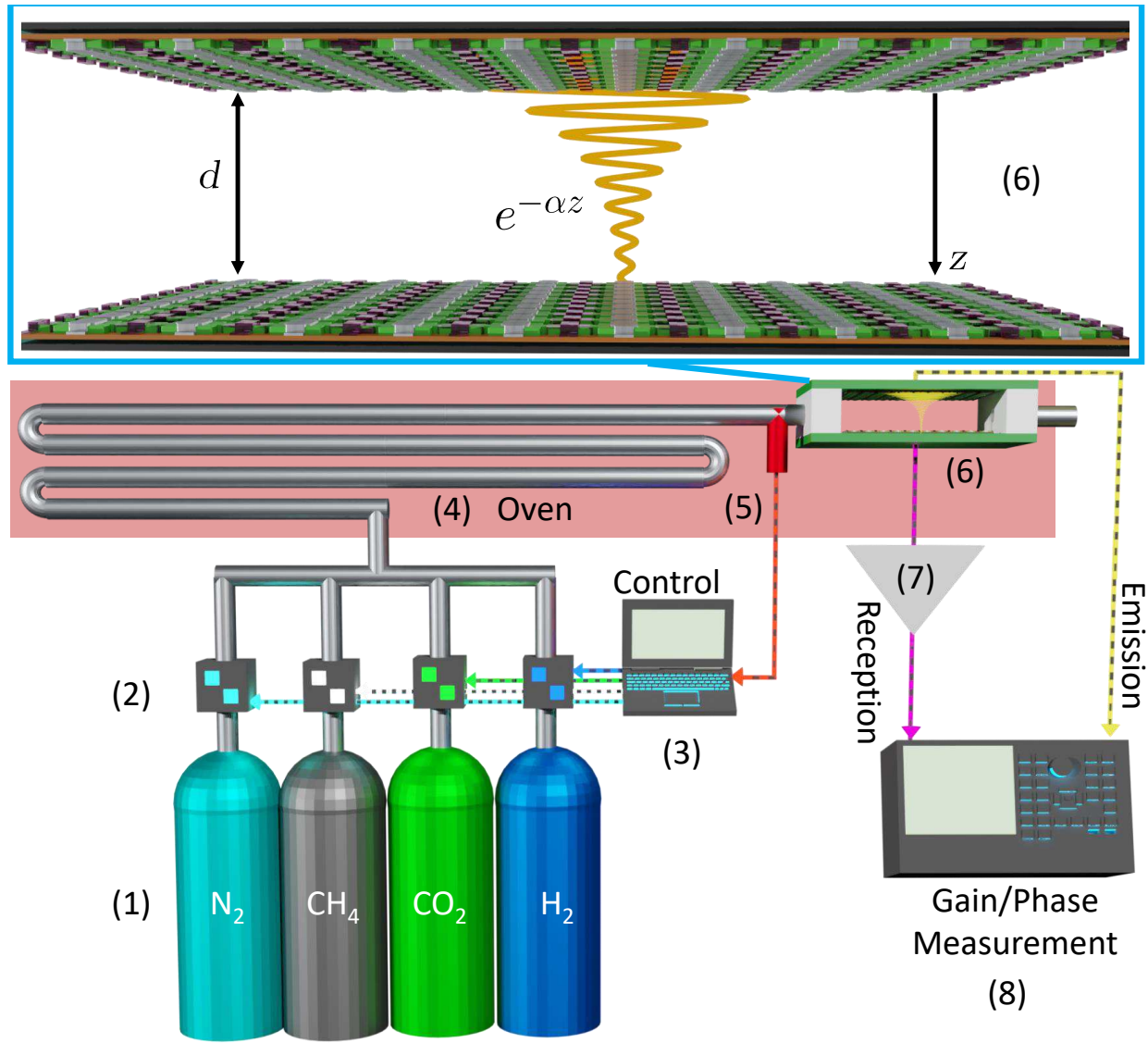

Figure 5. Complete setup schematics. (1) Gas bottles, (2) flowmeters, (3) flow and temperature control computer, (4) oven, (5) thermocouple, (6) measurement cell with zoom at the top of the image, (7) charge amplifier, (8) gain/phase network analyzer.

(3) at the desired concentrations. The mixture passes through an oven (4) where the desired temperature is set and verified by a thermocouple (5). The gas enters the measurement cell (6) where a continuous ultrasonic sine wave is sent from an emitter CMUT array to a receiver CMUT array after travelling a distance $d$. Thus, the wave arrives attenuated exponentially with $d$. Additionally its phase is shifted with respect to the emission by an amount proportional to the time of flight, $\tau=d / v$, required to go through the measuring cell. The receiver is connected to a charge amplifier (7) in order to improve the signal to noise ratio before being fed along with the emission signal to a gain/phase network analyzer (8).

\subsection{No Reflections Model}

Far from the resonance of the CMUTs, on the side of lower frequencies, one can assume that their electromechanical transfer function is a constant. Thus, using the complex formalism, the pressure wave $p$ can be considered of the form:

$$
p(t, z)=P_{00} e^{-\alpha z} e^{j(\omega t-k z)},
$$


where $P_{00}$ is the amplitude of the wave at $t=0$ on the emission end, $\omega$ the excitation angular frequency, $j=\sqrt{-1}, t$ the time, $z$ the coordinate orthogonal to the CMUT array (Fig. 5), $\alpha$ the attenuation coefficient of the gas and $k$ the wave number which, in the case of a plane wave, is linked to the gas sound velocity $v$ as follows:

$$
k=\frac{\omega}{v} .
$$

Since the wave is continuous, at any given time $t_{0} \geq \tau$, the value of $p$ at the receiving end $(z=d)$ is:

$$
p\left(t_{0}, d\right)=P_{00} e^{-\alpha d} e^{j \omega t_{0}} e^{-j \omega d / v} .
$$

Thus, its phase is shifted by a value of $-\omega \tau(\tau=d / v)$ with respect to the value of $p$ at the emission end at the same time $t_{0}$ :

$$
p\left(t_{0}, 0\right)=P_{00} e^{j \omega t_{0}} .
$$

The transfer function due only to the setup, $H_{0}$, independent of the gas can be defined as:

$$
H_{0}=\left|H_{0}\right| e^{j \phi_{0}},
$$

where $\phi_{0}$ is a constant and accounts for the shifts due to the setup and $\left|H_{0}\right|$ is the modulus of $H_{0}$, which depends only on the angular frequency. Therefore the total phase shift $\phi$ is:

$$
\phi=-\omega \tau+\phi_{0},
$$

As a consequence $\tau$ is simply the slope of the curve $\phi(\omega)$. Additionally, the change in amplitude is simply $e^{-\alpha d}$. Hence the total gain $|H|$ is given by:

$$
|H|=\left|H_{0}(j \omega)\right| e^{-\alpha d},
$$

In order to overcome the need of knowing $\left|H_{0}\right|$, a calibration step was performed to measure $|H|$ under $\mathrm{N}_{2},|H|_{N 2}$. Indeed, the shift in attenuation with respect to $\mathrm{N}_{2}, \Delta \alpha$, is given by:

$$
\Delta \alpha=\frac{1}{d} \ln \frac{|H|_{N 2}}{|H|},
$$

\subsection{Dealing with multiple reflections}

In order to measure a high signal to noise ratio, especially for higher frequencies, it is possible to increase the excitation amplitude. However, this will cause reflections that will interfere with the main emission, particularly for lower frequencies, where the attenuation is smaller. Therefore, they will modify both $\phi$ and $|H|$. This section seeks to understand the extent of this effect and, particularly, how to retrieve information about both $\alpha$ and $\tau$. Assuming normal incidence and a reflection coefficient of $r$ identical for both CMUT arrays, at a given position $z$ and time $t$, the acoustic wave in the cell is the superposition of a wave travelling forward $p_{f}$ (towards increasing $z$ ) and one travelling backwards $p_{b}$ (towards decreasing $z$ ). The former is the sum of all the reflections that happen an even number of times ( 0 included) between the emission time and the measurement time i.e.:

$$
p_{f, n}=P_{00} e^{j \omega t} \sum_{i=0}^{n} r^{2 i} e^{-(j k+\alpha)(2 i d+z)},
$$

where $n$ is the echo order (initial 0). Similarly, $p_{b}$ is the sum of the waves that have been reflected an odd number of times.

$$
p_{b, n}=P_{00} e^{j \omega t} \sum_{i=1}^{n} r^{2 i-1} e^{-(j k+\alpha)(2 i d-z)} .
$$

Notice that the term of order 0 is not to be accounted since the emission happens only towards the increasing $z$. Both $p_{f}$ and $p_{b}$ are geometric sums of same quotient $q$ given by:

$$
q=r^{2} e^{-2 d(j k+\alpha)} .
$$

Since $|q|<1, p_{f}$ and $p_{b}$ are simply given by:

$$
p_{f, n}=p_{f, 0} \frac{1-q^{n+1}}{1-q}
$$




$$
p_{b, n}=u_{0}\left(\frac{1-q^{n+1}}{1-q}-1\right)
$$

where

$$
\begin{aligned}
& p_{f, 0}=P_{00} e^{j(\omega t-k z)-\alpha z} \\
& u_{0}=\frac{1}{r} P_{00} e^{j(\omega t+k z)+\alpha z} .
\end{aligned}
$$

The total acoustic signal is finally

$$
p_{n}=p_{f, n}+p_{b, n} .
$$

One can verify that, the case $n=0$ corresponds exactly to the model presented in Sec. 4.3. In permanent regime, one can consider the number of echoes to be infinite, resulting in the final acoustic signal $p_{\infty}$ :

$$
p_{\infty}(z)=\frac{p_{f, 0}(z)+q u_{0}(z)}{1-q} .
$$

The total transfer function $H$ is then given by:

$$
H=-H_{0} \frac{p_{\infty}(z=d)}{P_{00}}
$$

which equals:

$$
H=-H_{0} \frac{(r+1) e^{-j \omega \tau-\alpha d}}{\left(r e^{-j \omega \tau-\alpha d}-1\right)\left(r e^{-j \omega \tau-\alpha d}+1\right)}
$$

It should be noticed once again that without any reflections $(r=0) H(j \omega)$ is equal to the transfer function with a single peak:

$$
H_{n=0}=H_{0} e^{-j \omega \tau-\alpha d} .
$$

In order to extract information about $\alpha$ some additional calculations are needed. Indeed, by using the fact that for $|y|<1$ :

$$
\frac{1}{1-y}=\sum_{n=0}^{\infty} y^{n}
$$

and some basic series manipulations Eq. 29 can be rewritten as:

$$
H=\sum_{n=1}^{\infty} P_{n} e^{-j \omega(2 n-1) \tau},
$$

where

$$
P_{n}=H_{0}(r+1) r^{2(n-1)} e^{-(2 n-1) \alpha d} .
$$

It is important to point out that all the $P_{n}$ are independent of $\tau$. From this form it can be seen that $H$ is the sum of complex exponentials modulated by the functions $P_{n}$. Applying the inverse Fourier transform to $H$ (noted $\hat{H}$ ) defined as:

$$
\hat{H}=\int_{-\infty}^{\infty} H(f) e^{2 \pi j f t} d f,
$$

will result in:

$$
\hat{H}(t)=\sum_{n=1}^{\infty} \hat{P}_{n}(t-(2 n-1) \tau),
$$


where $\hat{P}_{n}$ is the inverse Fourier transform of $P_{n}$. Therefore, $\hat{H}$ is the sum of peaks, where the envelope is the shifted inverse Fourier transform of $P_{n}$. In particular, the amplitude of the second peak is given by:

$$
P_{1}=H_{0}(r+1) e^{-\alpha d} .
$$

Therefore, by isolating $\left|P_{1}\right|$ it is possible to apply the same formula from the single reflection case (Eq. 18) to retrieve $\Delta \alpha$. Fig. 6.a shows the measured result of Eq. 34 under pure $\mathrm{N}_{2}$ as a function of the delay with respect to the emission, $t$, which is consistent with Eq. 35. It should be noted that, apart from the main peak $\hat{P}_{1}$ (at $t=\tau$ ) and the reflected echo $\hat{P}_{2}$ (at $\left.t=3 \tau\right)$ there is an additional peak at $t=0$. This is due to an instantaneous electrical coupling between the emitter and the receiver resulting from the high voltages applied ${ }^{17}$. However, since the objective is to isolate $P_{1}$, the coupling won't influence the final result. Fig. 6.b shows the wavelet transform of the measurement from Fig. 6.a and shows that the main peak contains more spectral information, since the highest frequencies are no longer present in the reflected echo. This supports the fact that in order to have information about the attenuation at the highest frequencies it is useful, and sometimes necessary, to accept the presence of reflections of the lower frequency components constituting the acoustic wave. However, this perturbs strongly $H$. This can be appreciated in Fig. 6.c and 6.d, which show the comparison between the measured transfer function (both with multiple reflections and electrical coupling) and the result of the data processing after retrieving only the contribution of $P_{1}$ by filtering out components of $\hat{H}$, which are outside of the range $t \in[15,30] \mu \mathrm{s}$. This numerical criterion should be modified according to the application ( $v$ and $d$ ). In our case, $\tau$ is not expected to vary more than $1 \mu$ s. It should be noted that, after filtering, $|H|$ is smooth as one would expect from the case without reflections. Moreover, the unwrapped ( $2 \pi$-modulo removed) phase after filtering is consistent with the model of a single peak as shown in Sec. 2 its slope corresponds to $-2 \pi \tau$.
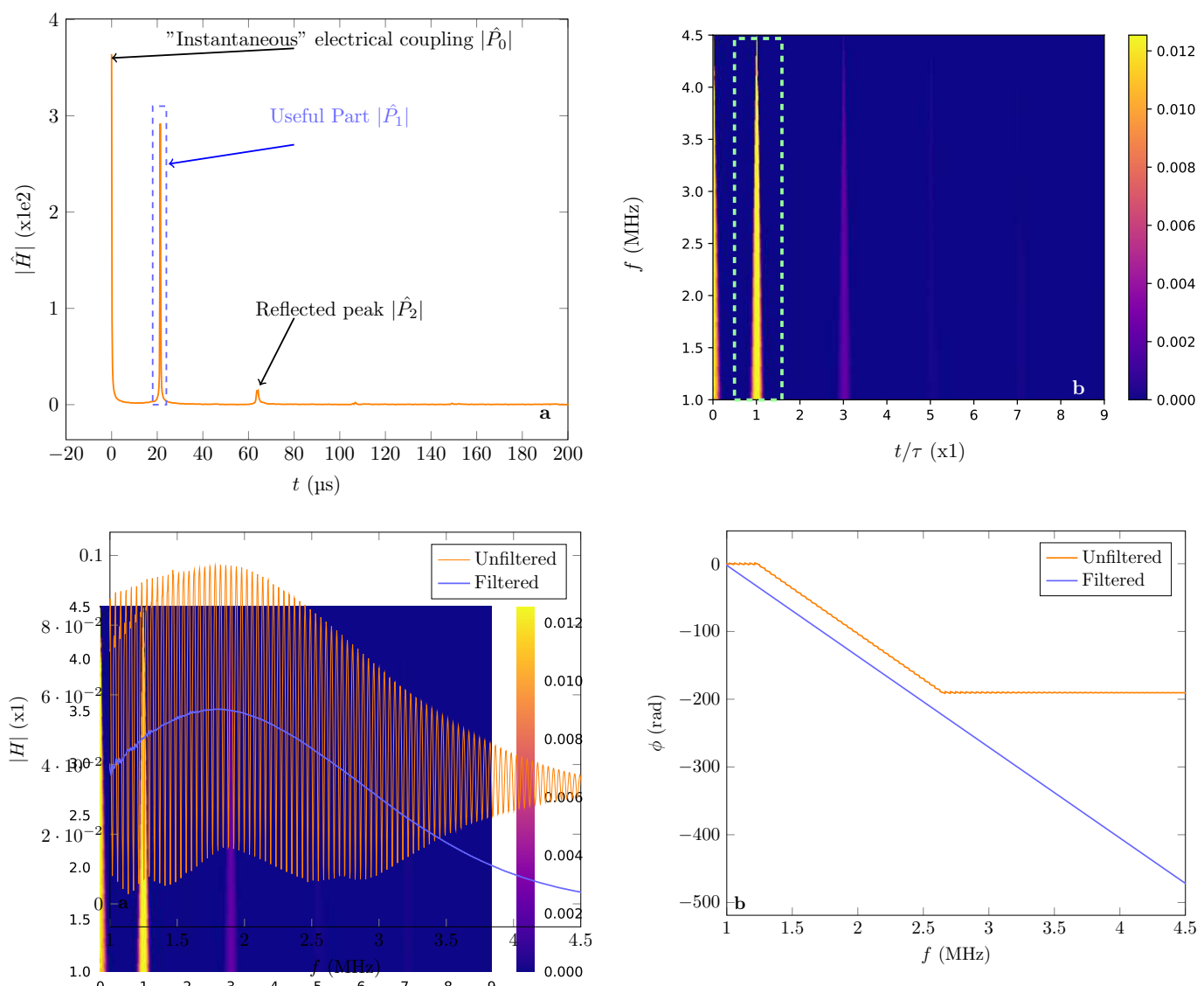

Figure 6. (a) Measurement of $|\hat{H}|$ as a function of the delay with respect to the emission. (b) Its wavelet transform diagram. (c) and (d) Comparison between the transfer function for multiple reflections (unfiltered) and after the main echo is extracted (filtered) for the gain and phase, respectively. 


\section{References}

1. Abdul, S., Judit, T., Ilona, F. \& Nikoletta, M. Functional thin films and nanostructures for sensors. In Fundamentals of Nanoparticles, 485-519 (Elsevier, 2018). URL elsevier.com/retrieve/pii/B9780323512558000161.

2. Chauhan, P. S. \& Bhattacharya, S. Hydrogen gas sensing methods, materials, and approach to achieve parts per billion level detection: A review. International Journal of Hydrogen Energy S0360319919330034 (2019). URL elsevier. com/retrieve/pii/s0360319919330034.

3. Sharma, R. K. et al. Investigation of stability and reliability of tin oxide thin-film for integrated micro-machined gas sensor devices. Sensors and Actuators B: Chemical 81, 9-16 (2001). URL elsevier.com/retrieve/pii/ S0925400501009200.

4. Hossein-Babaei, F. \& Ghafarinia, V. Compensation for the drift-like terms caused by environmental fluctuations in the responses of chemoresistive gas sensors. Sensors and Actuators B: Chemical 143, 641-648 (2010). URL elsevier. com/retrieve/pii/s0925400509007746.

5. Valcárcel, M. et al. Optimization of electronic nose drift correction applied to tomato volatile profiling. Analytical and Bioanalytical Chemistry (2021). URL springer.com/10.1007/s00216-021-03340-5.

6. Sader, J. E. Frequency response of cantilever beams immersed in viscous fluids with applications to the atomic force microscope. Journal of Applied Physics 84, 64-76 (1998). URL a ip. scitation.org/doi/10 .1063/1.368002.

7. Everest, F. A. The master handbook of acoustics (McGraw-Hill, New York, 2001), 4th ed edn.

8. Ejakov, S. G., Phillips, S., Dain, Y., Lueptow, R. M. \& Visser, J. H. Acoustic attenuation in gas mixtures with nitrogen: Experimental data and calculations. The Journal of the Acoustical Society of America 113, 1871-1879 (2003). URL asa.scitation.org/doi/10.1121/1.1559177.

9. Liu, T., Hu, Y., Zhang, X. \& Zhu, M. Acoustic analysis of gas compositions based on molecular relaxation features. Results in Physics 25, 104304 (2021). URL elsevier.com/retrieve/pii/s2211379721004344.

10. Rosario, R. \& Mutharasan, R. Piezoelectric excited millimeter sized cantilever sensors for measuring gas density changes. Sensors and Actuators B: Chemical 192, 99-104 (2014). URL elsevier.com/retrieve/pii/ S0925400513012033.

11. Boudjiet, M. et al. Geometry optimization of uncoated silicon microcantilever-based gas density sensors. Sensors and Actuators B: Chemical 208, 600-607 (2015). URL elsevier.com/retrieve/pii/s 0925400514014476.

12. Suchenek, M. \& Borowski, T. Measuring Sound Speed in Gas Mixtures Using a Photoacoustic Generator. Int J Thermophys 39, 11 (2018). URL springer.com/10.1007/s10765-017-2335-2.

13. Iglesias, L. et al. Proof of Concept and Preliminary Results of Gas Detection by Measuring the Admittance at the Resonance and Anti-resonance of an Uncoated CMUT. Front. Mech. Eng. 6, 14 (2020). URL www. frontiersin. org/article/10.3389/fmech.2020.00014/full.

14. Hök, B., Blückert, A. \& Löfving, J. Acoustic gas sensor with ppm resolution. Sensor Review 20, 139-142 (2000). URL www.emerald.com/insight/content/doi/10.1108/02602280010319231/full/html.

15. Iglesias, L. et al. Hydrogen Selective Gas Sensor Based on Attenuation Measurement of Acoustic Wave Using Uncoated CMUT. In 2021 IEEE 34th International Conference on Micro Electro Mechanical Systems (MEMS), 398-401 (IEEE, Gainesville, FL, USA, 2021). URL ieeexplore. ieee. org/document/9375432/.

16. Iglesias, L., Boudjiet, M. \& Dufour, I. Discrimination and concentration measurement of different binary gas mixtures with a simple resonator through viscosity and mass density measurements. Sensors and Actuators B: Chemical 285, 487-494 (2019). URL elsevier.com/retrieve/pii/S0925400519300978.

17. Shanmugam, P. et al. Broad bandwidth air-coupled micromachined ultrasonic transducers for gas sensing. Ultrasonics 114, 106410 (2021). URL elsevier.com/retrieve/pii/s0 041624 X21000512.

18. Iglesias, L. et al. CMUT Time of Flight Gas Sensor By Phase Shift Measurement. IMCS MA2020-01, 2323-2323 (2020). URL iopscience.iop.org/article/10.1149/MA2020-01312323mtgabs.

19. Törndahl, M., Almqvist, M., Wallman, L., Persson, H. \& Lindstrom, K. Characterisation and comparison of a cMUT versus a piezoelectric transducer for air applications. In 2002 IEEE Ultrasonics Symposium, 2002. Proceedings., vol. 2, 1023-1026 (IEEE, Munich, Germany, 2002). URL ieeexplore. ieee.org/document/1192469/.

20. Graves, R. E. \& Argrow, B. M. Bulk Viscosity: Past to Present. Journal of Thermophysics and Heat Transfer 13, 337-342 (1999). URL arc.aiaa.org/doi/10.2514/2.6443. 
21. Zhang, K. et al. Calculating vibrational mode contributions to sound absorption in excitable gas mixtures by decomposing multi-relaxation absorption spectroscopy. Applied Acoustics 116, 195-204 (2017). URL elsevier. com/retrieve/ pii/s0003682X16303036.

22. Law, A. K., Koronaios, N. \& Lindsay, R. B. Effect of Diffusion on Ultrasonic Attenuation in Mixtures of the Rare Gases. The Journal of the Acoustical Society of America 41, 93-99 (1967). URL asa . scitation.org/doi/10.1121/1. 1910336.

23. Heller, J., Boulme, A., Alquier, D., Ngo, S. \& Certon, D. Performance Evaluation of CMUT-Based Ultrasonic Transformers for Galvanic Isolation. IEEE Transactions on Ultrasonics, Ferroelectrics, and Frequency Control 65, 617-629 (2018). URL ieeexplore. ieee.org/document/8265184/.

24. Engineering ToolBox I www.engineeringtoolbox.com/ (June 2020).

25. Cramer, M. S. Numerical estimates for the bulk viscosity of ideal gases. Physics of Fluids 24, 066102 (2012). URL aip.scitation.org/doi/10.1063/1.4729611.

26. Wang, Y., Ubachs, W. \& van de Water, W. Bulk viscosity of $\mathrm{CO}_{2}$ from Rayleigh-Brillouin light scattering spectroscopy at 532 nm. J. Chem. Phys. 150, 154502 (2019). URL aip. scitation. org/doi/10.1063/1.5093541.

27. Zuckerwar, A. J. \& Griffin, W. A. Effect of water vapor on sound absorption in nitrogen at low frequency/pressure ratios. The Journal of the Acoustical Society of America 69, 150-154 (1981). URL asa.scitation.org/doi/10.1121/ 1.385359.

28. Winter, T. G. \& Hill, G. L. High Temperature Ultrasonic Measurements of Rotational Relaxation in Hydrogen, Deuterium, Nitrogen, and Oxygen. The Journal of the Acoustical Society of America 42, 848-858 (1967). URL asa . scitation . org/doi/10.1121/1.1910657.

29. Higgins, B. G. \& Binous, H. Binary diffusion coefficients for gases I demonstrations.wolfram.com/binarydiffusioncoefficientsforgases/ (June 2020).

30. Bogatyrev, A. F., Belalov, V. R. \& Nezovitina, M. A. Thermal diffusion in binary mixtures of moderately dense gases. $J$ Eng Phys Thermophy 86, 1225-1231 (2013). URL springer.com/10.1007/s10891-013-0945-5.

31. Maghari, A. \& Hossain Jalili, A. Calculation of Transport Coefficients for $\mathrm{CH}_{4}-\mathrm{N}_{2}$ and $\mathrm{CH}_{4}-\mathrm{O}_{2}$ by the Inversion Method. J. Phys. Soc. Jpn. 73, 1191-1196 (2004). URL journals. jps. jp/doi/10.1143/JP S . 73.1191.

32. Trengove, R. D., Robjohns, H. L. \& Dunlop, P. J. Diffusion Coefficients and Thermal Diffusion Factors for the Systems H2-N2, D2-N2, H2-O2 and D2-O2. Berichte der Bunsengesellschaft f̃̃ $1 / 4$ r physikalische Chemie 87, 1187-1190 (1983). URL doi.wiley.com/10.1002/bbpc.19830871220.

\section{Acknowledgements}

This research was performed thanks to the contest Programme d'Investissements d'Avenir of the French Goverment under the supervision of the French National Radioactive Waste Management Agency (ANDRA).

\section{Authors' contributions}

PS, J-FM, DA and DC have designed and fabricated the CMUTs. LI and ID have developed the modeling and data treatments. LI has done the measurements. All the authors have contributed to the writing of the paper.

\section{Competing interests}

The authors declare no conflict of interests. 